Journal of Computer Science 8 (10): 1644-1648, 2012

ISSN 1549-3636

(C) 2012 Science Publications

\title{
Reactive Power Optimization Using Quantum Particle Swarm Optimization
}

\author{
${ }^{1}$ Chandragupta Mauryan, K.S., ${ }^{2}$ K. Thanushkodi and ${ }^{3}$ A. Sakthisuganya \\ ${ }^{1}$ Department of Electrical and Electronics, \\ Sri Krishna College of Technology, Coimbatore, Tamilnadu, India \\ ${ }^{2}$ Akshaya College of Engineering and Technology, Coimbatore, Tamilnadu, India \\ ${ }^{3}$ Department of Electrical and Electronics, \\ Sri Krishna College of Technology, Coimbatore, Tamilnadu, India
}

\begin{abstract}
Problem statement: The problem of controlling a power system is not an easy task; it is subjected to various constraints. There are at risks of voltage instability problems due to highly stressed operating conditions caused by increased load demand and other constraints in the power system network. Approach: This study presents the implementation of Quantum Particle Swarm Optimization (QPSO) in solving the Reactive Power Optimization (RPO) problem. The main aim of this algorithm is the minimization of the real power loss and to improvise the voltage in the system. In this new algorithm, the particles were made to perform studies on itself and also the best ones in the system. Results: The implementations of QPSO were carried on modified IEEE 14 bus system for obtaining solution to the reactive power optimization and the output results are found predominant with classical PSO. Conclusion: This technique is used to find the best solution and also the convergence time is reduced. The proposed QPSO method is demonstrated and results are compared with traditional optimization methods.
\end{abstract}

Key words: Quantum Particle Swarm Optimization (QPSO), optimal reactive power control, Reactive Power Optimization (RPO), conditions caused, found predominant

\section{INTRODUCTION}

The main need of Reactive Power Optimization and Voltage Control is to improvise the voltage stability in the system and to minimize the losses. The power systems are provided with a number of voltage controlling devices such as generators, tap changing transformers, shunt capacitors/reactors, synchronous condensers and static VAR compensators etc. By varying the load or network configuration, a real time control of these devices will increase the problem. The conventional PSO can handle only continuous controls. QPSO method can be easily extended to handle discrete controls. This paper describes about QPSO algorithm and its application to RPO problem considering both continuous and discrete controls (Ciuprina et al., 2002).

Optimal reactive power control problem: The primary objective of Reactive Power Optimization is to reduce system power losses and to obtain the setting of various controls, for the same. The real power loss is a non-linear function of bus voltage and its corresponding phase angles.It is a function of its control variables (Ning et al., 2007) Eq. 1:

$$
\operatorname{MinP}_{\mathrm{L}}=\sum \mathrm{G}_{\mathrm{K}}\left[\mathrm{V}_{\mathrm{i}}^{2}+\mathrm{V}_{\mathrm{j}}^{2}-2 \mathrm{~V}_{\mathrm{i}} \mathrm{V}_{\mathrm{j}} \cos \left(\alpha_{\mathrm{i}}-\alpha_{\mathrm{j}}\right)\right]
$$

The number of lines in the network is denoted by $\mathrm{K}$. The above function involves various constraints for its minimization. Eq. 2-4:

$$
\begin{aligned}
& 0=\mathrm{P}_{\mathrm{gi}}-\mathrm{P}_{\mathrm{di}}-\mathrm{V}_{\mathrm{i}} \sum \mathrm{V}_{\mathrm{j}}\left(\mathrm{G}_{\mathrm{ij}} \cos \theta_{\mathrm{ij}}+\mathrm{B}_{\mathrm{ij}} \sin \theta_{\mathrm{ij}}\right) \\
& 0=\mathrm{Q}_{\mathrm{gi}}-\mathrm{Q}_{\mathrm{di}}-\mathrm{V}_{\mathrm{i}} \sum \mathrm{V}_{\mathrm{j}}\left(\mathrm{G}_{\mathrm{ij}} \sin \theta_{\mathrm{ij}}+\mathrm{B}_{\mathrm{ij}} \cos \theta_{\mathrm{ij}}\right) \\
& \mathrm{Q}_{\mathrm{ci}} \min <\mathrm{Q}_{\mathrm{ci}}<\mathrm{Q}_{\mathrm{ci}} \max _{\mathrm{i} \in \mathrm{n}_{\mathrm{C}}} \\
& \mathrm{Q}_{\mathrm{gi}} \min <\mathrm{Q}_{\mathrm{ci}}<\mathrm{Q}_{\mathrm{ci}} \max _{\mathrm{i} \in \mathrm{n}_{\mathrm{g}}} \\
& \mathrm{T}_{\mathrm{k}} \min <\mathrm{T}_{\mathrm{k}}<\mathrm{T}_{\mathrm{k}} \max _{\mathrm{k} \in \mathrm{n}_{\mathrm{c}}} \\
& \mathrm{V}_{\mathrm{i}} \min <\mathrm{V}_{\mathrm{i}}<\mathrm{V}_{\mathrm{i}} \max _{\mathrm{i} \in \mathrm{n}}
\end{aligned}
$$

Here $\mathrm{n}$ is the total number of buses, $\mathrm{n}_{\mathrm{g}}$ and $\mathrm{n}_{\mathrm{c}}$ are number of generator and reactive sources, $n_{t}$ are the number of tap changers. Power flow Eq. 2 and 3 are used as equality constraints, reactive power source

Corresponding Author: Chandragupta Mauryan, K.S., Department of Electrical and Electronics, Sri Krishna College of Technology, Coimbatore, Tamilnadu, India 
installation restrictions, reactive generation restrictions, transformer tap-setting restrictions, bus voltage restrictions (4) are used as inequality constraints (Sun et al., 2005; Platel et al., 2009).

\section{MATERIALS AND METHODS}

Insight of particle swarm optimization: Particle Swarm Optimization is one of the computation technique to solve RPO problem.It is based on the concept of social behavior of organsims like school of fish etc. A flock of birds has its own objective function. (Wong and Dong, 2008). Each particle knows its own best value (pbest) and its XY position.Each particle knows the best value in their group known as gbest.Based on their velocity and distance between their best values (pbest and gbest), their position can be attained and modified. The velocity of each particle represents their modification. The velocity is modified with resepct to the following equation. Eq. 5:

$\mathrm{V}^{\mathrm{k}+1} \mathrm{w}_{\mathrm{i}} \mathrm{V}^{\mathrm{k}}+\mathrm{c}_{1} \mathrm{r}_{1}\left(\right.$ pbest $\left._{\mathrm{i}}-\mathrm{X}_{\mathrm{i}}^{\mathrm{k}}\right)+\mathrm{c}_{2} \mathrm{r}_{2}\left(\right.$ gbest $\left._{\mathrm{i}}-\mathrm{X}_{\mathrm{i}}^{\mathrm{k}}\right)$

Where:

$\mathrm{V}^{\mathrm{k}} \quad=$ Current velocity of the particle $\mathrm{i}$ at iteration $\mathrm{k}$

$\mathrm{V}^{\mathrm{k}+1}=$ Modified velocity of particle

$\mathrm{r}_{1}, \mathrm{r}_{2}=$ Random numbers between 0 and 1

$\mathrm{X}_{\mathrm{i}}^{\mathrm{k}}=$ Current position of particle $\mathrm{i}$ at iteration $\mathrm{k}$

Pbest $_{i}=$ Pbest of particle

Gbest $_{i}=$ Gbest of the group

$\mathrm{w}_{\mathrm{i}}=$ Weight function for velocity of particle

$\mathrm{c}_{\mathrm{i}}=$ Weight coefficients for each term

From the above equation a certain velocity that gets close to pbest and gbest is calculated. The current position of the particel can be modified by the following Eq. 6 :

$$
X_{i}^{k+1}=X_{i}^{K}+V^{k+1}
$$

This search procedure is called as Classical Particle Swarm Optimization (Classical PSO).

Characteristics of QPSO: The primary objective of RPO problem is reduction in the system power losses and to obtain the setting of various controls, for the same. The real power loss is a non-linear function of bus voltage magnitudes and phase angles. Based on the problem of traditional PSO trapping into local optima, Quantum theory is introduced into PSO to strengthen the particle diversities and to avoid the premature convergence.The QPSO algorithm has better convergence characteristics than the classical method.
Table 1: Comparison of QPSO and classical PSO

\begin{tabular}{lcr}
\hline Variable & Classical PSO & \multicolumn{1}{c}{ QPSO } \\
\hline Voltage & 1.0300 & 0.97 \\
No. of iteration & 75.0000 & 50.00 \\
Time taken & 15.7000 & 4.50 \\
Power loss & 81.4657 & 68.3213 \\
\hline
\end{tabular}

Table 2: Power loss with respect to iteration

\begin{tabular}{lllc}
\hline $\begin{array}{l}\text { Iteration } \\
\text { Time }(\mathrm{sec})\end{array}$ & $\begin{array}{l}\text { Generation } \\
\text { Count }\end{array}$ & $\begin{array}{l}\text { PQ Generated } \\
\text { power }(\mathrm{MW})\end{array}$ & $\begin{array}{l}\text { Ploss } \\
(\mathrm{MW})\end{array}$ \\
\hline 0.64073 & 30 & 2000 & 13.4 \\
0.62822 & 53 & 3200 & 12.8 \\
0.63766 & 54 & 4500 & 11.0 \\
0.62974 & 85 & 5400 & 10.2 \\
0.64577 & 92 & 6900 & 7.3 \\
0.63364 & 110 & 7600 & 7.5 \\
0.00017 & 115 & 8550 & 3.2 \\
\hline
\end{tabular}

The power loss and the number of iterations required to recah optimal value are compaaratively less than the classical approach.The comparative results between classical PSO and QPSO is represented in Table 1. This algorithm shows that it has better convergence, stronger optimal ability and better global searching capability than PSO (Pant et al., 2007).

Quantum computing is a newly introduced theory based on the concepts from quantum mechanics and computer science. The origin of quantum computing dates back to the 80's when Richard Feynman found that certain quantum mechanical results cannot be effectively simulated on a personal computer. During 1990's , quantum computing has attracted worldwide interest and has motivated researchers to work on it, as it appears more effective and efficient than the traditional methods. One of the striking feature of QPSO is parallel processing which can be used to solve combinatorial optimization problems and reduces the complexity.(Layeb and Saidouni 2007).

The Quantum inspired Particle Swarm Optimization (QPSO) is a recent optimization technique which is based on quantum mechanics. Similar to an evolutionary algorithm, QPSO also relies on the representation of the individual, the evaluation function and the population dynamics. QPSO represents the individual for a given problem and finally solution can be obtained.The RPO problem can be effectively solved by using this technique. The power loss with respect to the iteration is shown in Table 2. From the table we can find the Generation count for powerloss and the power generated for each iteration.

Formulation of RPO problem: In order to use QPSO algorithm to solve the RPO problem, the following control variables are considered:

- Generator reactive powers

- Generator voltage magnitudes 
Of the above controls considered, the Generator reactive powers and voltage magnitudes are considered as continuous, where as the tap settings of transformers and shunt reactive sources are considered as discrete controls. So, the particles position vector is given by Eq.7:

$$
\left.\mathrm{X}=\mathrm{Q} 1, . . \mathrm{Qn}_{\mathrm{g}}, \mathrm{v1}, . . \mathrm{vn} \mathrm{g}_{\mathrm{g}}, \mathrm{t} 1, . . \mathrm{tn}_{\tau}, \mathrm{Q} 1, . . \mathrm{Qn}_{\mathrm{c}}\right)
$$

Where:

$\mathrm{n}_{\mathrm{g}}=$ Is no. of generator buses

$\mathrm{n}_{\mathrm{t}} \quad=$ Is no. of tap settings

$\mathrm{n}_{\mathrm{c}}=$ Is no. of shunt reactive sources

The steps using QPSO to solve loss control problem is represented here. The pseudo code of the procedure is as follows (Liu et al., 2006; 2005):

- For each particle, initialize quantum particle of the swarm(population).

- Calculate the mean best (mbest) from the equation

- Update the position of the particle in the swarm

- Update pbest value

- Update gbest value

- Continue the iteration till maximum criteria is reached.

The procedure for implementing the QPSO is given by the following steps (Sun et al., 2004).
Step1: Initialization of swarm positions: Initialize a population with random positions in the ndimensional problem space using a uniform probability distribution function.

Step2: Evaluation of particle's fitness. Comparison to pbest (personal best): Compare each particle's fitness with its pbest value.

Step3: Comparison to gbest (global best): Compare the fitness with the population's overall pbest and updating of global point: Calculate the Mbest

Step4: Repeating the evolutionary cycle: Loop to Step 2 until a stop criterion is reached.

\section{RESULTS AND DISCUSSION}

The proposed model has been applied to modified IEEE 14 bus system as shown in Fig 1. The QPSO algorithm is tested on modified IEEE 14 bus system and the results obtained are tabulated. The ORPC problem is solved by QPSO. The Particle represents the controls used in the ORPC problem. The result shows that there is optimized loss and controls with better convergence characteristic. The parameters considered are:

- Voltages of generators at Buses 6 and 5

- Tap settings of both transformers

- VAR's of shunt capacitors at Buses 1 and 3.

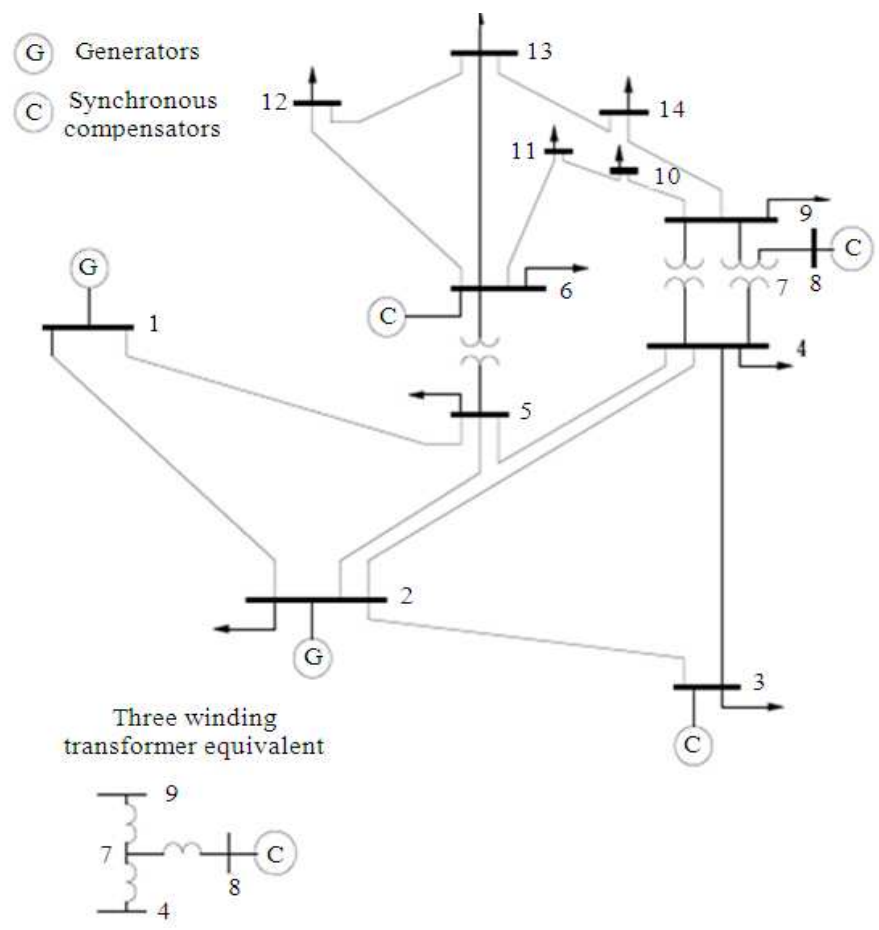

Fig. 1: Modified IEEE 14 bus system 


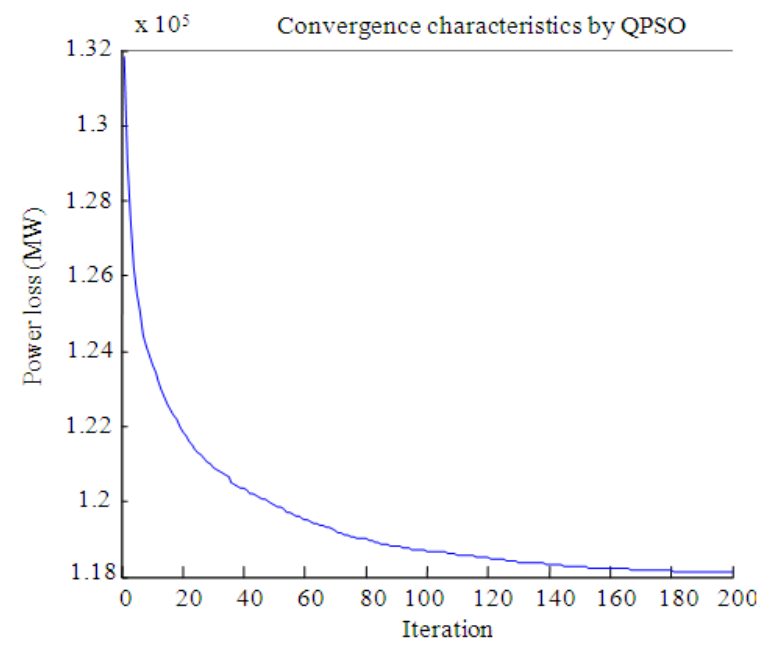

Fig. 2: Convergence characteristics of QPSO

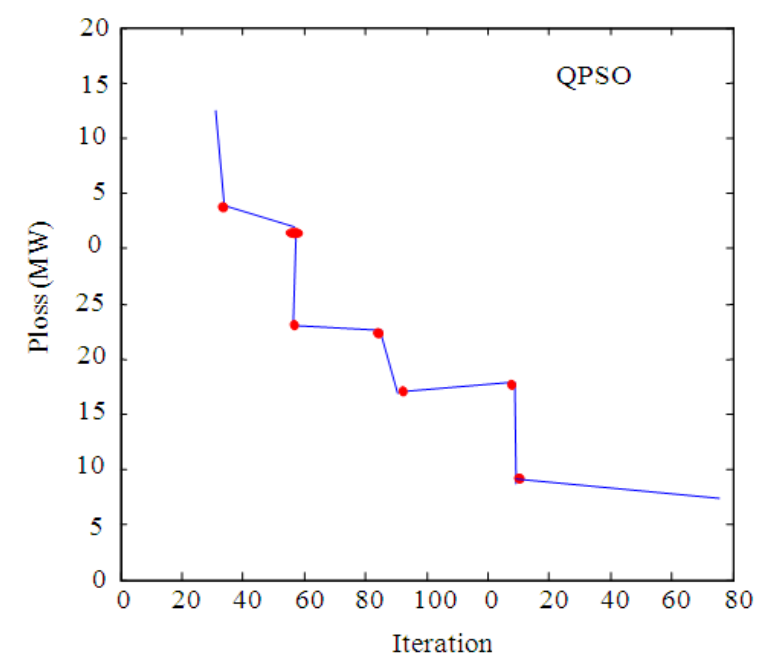

Fig. 3: Power loss characteristics by QPSO

Table 3: Comparison of the simulation results

\begin{tabular}{lrrr}
\hline Variable & Linear method & Classical PSO & \multicolumn{1}{c}{ QPSO } \\
\hline Reactive power & 223.456 & 217.666 & 207.431 \\
Generation reactive power & 44.567 & 39.126 & 36.635 \\
Loss total real power & 143.678 & 135.913 & 128.454 \\
Loss voltage & 1.560 & 1.020 & 0.96 \\
\hline
\end{tabular}

The comparison of various parameters like real power loss and voltage loss between QPSO and other conventional methods are reprsented in Table 3. From the table it shows that QPSO is highy efficient algorithm to reach global optimal value.The converegnce characteristics is shown in Fig 2 and the power loss characteristics are shown in Fig 3. It clearly depicts that the convergence rate is fast and hence QPSO is an effective technique for optimization.
The reactive power controlling devices are set to minimize the system real power losses and maintain the dependent variables within limits.

\section{CONCLUSION}

The developed algorithm has been tested on modified IEEE 14 bus system for ORPC. The results using QPSO and Classical PSO are compared. The numerical results show the competitiveness of developed QPSO over Classical PSO. In Classical PSO, in the initial stages the particles converge very quickly, however, as the iteration goes on, particles become very similar and almost have no ability to explore new areas. The results justify the fact that by considering the neighbouring particle the Global solution is enhanced and speed of convergence is achieved.

\section{REFERENCES}

Ciuprina, G., D. Ioan and I. Munteanu, 2002. Use of intelligent-particle swarm optimization in electromagnetics. IEEE Trans. Magne., 38: 10371040. DOI: $10.1109 / 20.996266$

Ning, Z.G., K. Meng, X.F. Yan and F. Qian, 2007. An improved particle swarm algorithm and its application in soft sensor modeling. J. East China Univ. Sci. Technol.,

Wong, K.P. and Z.Y. Dong, 2008. Differential Evolution, an Alternative Approach to Evolutionary Algorithm. In: Modern Heuristic Optimization Techniques: Theory and Applications to Power Systems, Lee, K. and M. El-Sharkawi, (Eds.), John Wiley Sons, Ltd, USA, pp: 171-187.

Pant, M., R. Thangaraj and A. Abraham, 2007. A new PSO algorithm with crossover operator for global optimization problems. Innovations Hybrid Intell. Syst., 44: 215-222. DOI: 10.1007/978-3-54074972-1_29

Sun, J., W. Xu and B. Feng, 2005. Adaptive parameter control for quantum-behaved particle swarm optimization on individual level. IEEE Int. Con. Sys. Man Cybernetics, 4: 3049-3054. DOI: 10.1109/ICSMC.2005.1571614

Platel, M.D., S. Schliebs and N. Kasabov, 2009. Quantum-inspired evolutionary algorithm: A multimodel EDA. IEEE Trans. Evolut. Comput., 13: 1218-1232. DOI: 10.1109/TEVC.2008.2003010

Layeb, A. and D.E. Saidouni, 2007. Quantum Genetic algorithm for binary decision diagram ordering problem. Int. J. Comput. Sci. Network Secu., 7: 130-135. 
Liu J., J. Sun and W. Xu, 2006. Quantum-behaved particle swarm optimization with adaptive mutation operator. Adv. Natural Comput., 4221: 959-967. DOI: $10.1007 / 11881070 \_126$

Liu J., W. Xu and J. Sun, 2005. Quantum-behaved particle swarm optimization with mutation operator. Proceedings of the 17th IEEE, International Conference Tools with Artificial Intelligence, Nov. 16-16, IEEE XPlore Press, Hong Kong, pp: 4-240. DOI: 10.1109/ICTAI.2005.104
Sun J., W. Xu and B. Feng, 2004. A global search strategy of quantum-behaved particle swarm optimization. Proceedings of the IEEE Conference on Cybernetics and Intelligent Systems, Dec. 1-3, IEEE Xplore Press, pp: 111-116. DOI: 10.1109/ICCIS.2004.1460396 